Mathematical Modelling and Analysis

Volume 21 Number 3, May 2016, 385-398

http://dx.doi.org/10.3846/13926292.2016.1172131

(c) Vilnius Gediminas Technical University, 2016
Publisher: Taylor\&Francis and VGTU

http://www.tandfonline.com/TMMA

ISSN: $1392-6292$

eISSN: 1648-3510

\title{
On a Planar Dynamical System Arising in the Network Control Theory*
}

\author{
Svetlana Atslega ${ }^{a}$, Dmitrijs Finaskins ${ }^{b}$ and \\ Felix Sadyrbaev ${ }^{c}$ \\ ${ }^{a, c}$ Institute of Mathematics and Computer Science, University of Latvia \\ Raiņa bulvāris 29, Rīga LV-1459, Latvia \\ ${ }^{b}$ Telecommunications institute, Riga Technical University \\ Azenes street 12, Rīga, LV-1048, Latvia \\ E-mail(corresp.): svetlana.atslega@llu.lv \\ E-mail: dmitrijs.finaskins@rtu.lv \\ E-mail: felix@latnet.lv
}

Received August 29, 2015; revised March 21, 2016; published online May 15, 2016

\begin{abstract}
We study the structure of attractors in the two-dimensional dynamical system $\frac{d x_{1}}{d t}=\frac{1}{1+e^{-\mu\left(x_{2}-\Theta\right)}}-x_{1}, \frac{d x_{2}}{d t}=\frac{1}{1+e^{-\mu\left(x_{1}-\Theta\right)}}-x_{2}$ that appears in the network control theory. We provide description of the attracting set and follow changes this set suffers under the changes of positive parameters $\mu$ and $\Theta$.
\end{abstract}

Keywords: dynamical system, attractor selection, phase portraits, networks control.

AMS Subject Classification: 34B15; 34B23; 34C60; 34D45.

\section{Introduction}

Core telecommunication networks nowadays are usually based on Wavelength Division Multiplexing (WDM) technology. In such division multiplexing technology transmitting/receiving channels are divided by wave length. It allows transmitting multiple traffic channels over a single fiber. Traffic by fiber optics can be transmitted over long distances without any additional equipment. This means that Internet Service Providers can save money by using fiber optics for transmitting big amount of traffic over long distances. This is why WDM networks are commonly used to carry Internet traffic at backbone Service Provider level. To route traffic over Internet the Internet Protocol (IP) is used. So there is need for a method of carrying IP traffic over WDM network. One possibility is to use Virtual Network Topology (VNT) approach for this purpose. This includes IP routers and optical fibers, transmitters/receivers. As was mentioned

* The support of the ESF project 2013/0024/1DP/1.1.1.2.0/13/ APIA/VIAA/045 is kindly acknowledged. 
above, a single fiber can carry a lot of traffic from different sources to different destinations by defining multiple channels, which are separated from each other by wave length.

There are multiple VNT control methods, which configure/reconfigure VNT according to traffic demand matrices in order to optimize network performance. Traffic demand matrices show how traffic flows are distributed via nodes and how these flows change over time. Nowadays Internet users are intensively using programs like Skype with video call opportunity, use online video services like YouTube or torrents, which generate a lot of traffic in unpredictable time moments. This causes constant and rapid changes in traffic flows between nodes (routers) in the network. There is need to reconfigure VNT over a period of time in order to provide high-level service with minimal delays. Thus VNT needs to be adaptable to changes in traffic demand. There are two modes of constructing traffic demand matrices, namely, offline and online or dynamic mode. In offline mode traffic demand matrices are constructed using previously known information about changes in traffic demand as it is described in [2], [3], [6], [11]. The major weakness of this approach is that offline methods do not work correctly if traffic flow changes are different from expectations. This will result in service quality drop.

Online approach allows reconfiguring VNT dynamically, no previous knowledge about traffic patterns is needed. In this case periodical measurement results or data received via well-known Simple Network Management Protocol (SNMP) could be used. To evaluate VNT status (performance), some information about network current state should be used like average or maximum link utilization, end-to-end packet delays. Based on this information new lightpaths can be added to source-destination pair of nodes if, for example, link utilization between these nodes exceeds the defined threshold and, consequently, can be removed if lightpaths currently used by the node pair are underutilized.

The majority of online VNT control methods are used when traffic demand is changing periodically and gradually [10]. This approach fails to work if changes of traffic demand are not predictable. This is the case in most real-life applications. So there is need to develop a VNT control method that is adaptable to unknown and unpredictable changes in network environment. This method should work also if these changes are rapid. Scientists and engineers tried to propose an algorithm that could successfully deal with all the above mentioned problems/limitations. Some researchers thought it could be a good idea to search for such an algorithm in Nature. It was noticed by biologists that cells in all living organisms are adaptable to unknown and unpredictable changes in environment. The used mechanism allows cells to adapt to changing environment even if these changes are very fast. This mechanism was described by [5]. It was proposed to use attractor selection, which represents mechanism of adaptation to unknown changes in biological systems [8], [7], [9]. The literature on gene regulatory systems is intensive and we would like to mention review articles [12], [1], [4].

The main idea of attractor selection is (accordingly to some authors such as $[9],[7],[8])$ that the system is driven by two components, namely, deterministic and stochastic. Attractors may consist of a set of the equilibrium points in the 
solution space. Conditions of such system are controlled by simple feedback. When conditions of a system are suitable (that is, close to one of the attractors), it is driven almost only by deterministic behavior and stochastic influence is limited. When conditions of the systems are poor, the deterministic behavior is negligible and in this case system is driven by stochastic behavior. In this case the system randomly fluctuates searching for a new attractor. When this attractor is found, the deterministic behavior again dominates over stochastic [9]. Such approach could be used to adapt VNT to changes in traffic demand as well.

If we use an attractor selection mechanism for management of network resources, first we should define the regulatory matrix $W$, which shows relationships between node pairs, that is, how any node pair affects another one including itself. As it was described in [5], there are three types of influence, namely, activation, inhibition and no relation, corresponding to $W_{i j}$ values of $1,-1$ and 0 .

The better approach to be used is to define the regulatory matrix $W$ based on network topology. With this we would ensure that matrix W reflects the correct relationships between node pairs, thus attractor selection method would provide optimal solution in terms of network management.

If we define matrix $W$ based on network topology, then activation (the respective $W_{i j}$ value is 1 ) would correspond to situations where setting-up additional lightpaths for the particular node pair would mean more effective traffic routing. As an example we could mention the situation when 3 nodes are connected in a series (Figure 1).

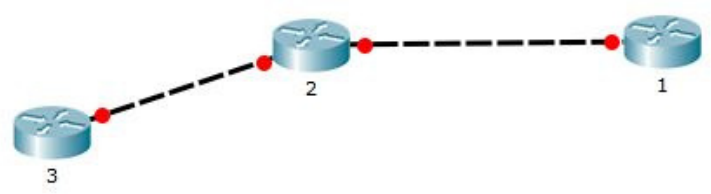

Figure 1. Two node pairs activate another node pair for more efficient routing.

Node pairs $1-2$ and $2-3$ should activate node pair $1-3$ to establish a direct link between nodes 1 and 3 and to avoid configuring lightpaths $1-2$ and $2-3$. This would ensure more effective lightpaths usage and reduce utilization of computing resources of Router 2. Inhibition (the corresponding $W_{i j}$ value is -1 ) would mean that node pairs are competing for shared resources. As an example such situation is visualized in Figure 2.

No relation (the respective $W_{i j}$ value is 0 ) corresponds to situations when node pairs are located in different parts of the network and do not interrelate. In this paper we will investigate the behavior of the network shown in Figure 2.

Since node pairs $1-2$ and $3-4$ are competing for the resources (lightpaths) between nodes 5 and 6 , these pairs will inhibit each other and the respective entries of matrix $W$ are -1 . We assume that traffic can flow only from node 1 


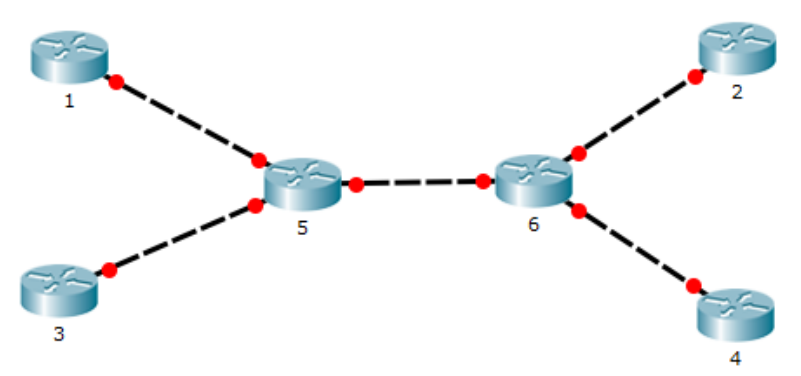

Figure 2. Two nodes pairs are competing for resources.

to node 2 or from node 3 to node 4 ; no other combinations are allowed. This will simplify the system of differential equations that describes the mechanism of attractor selection for this network. In fact, the system consists only of 2 equations, describing node pairs $1-2$ and $3-4$. The node pair $5-6$ does not carry an independent traffic, traffic between these nodes consists only from traffic between node pairs $1-2$ and $3-4$. So the direct lightpaths between node pairs $1-2$ and $3-4$ will be established. To simplify calculations, we will assume that self-influence is 0 . This means that matrix $W$ is as follows:

$$
W=\left(\begin{array}{cc}
0 & -1 \\
-1 & 0
\end{array}\right) \text { or } W=\left(\begin{array}{ll}
0 & 1 \\
1 & 0
\end{array}\right)
$$

Matrix $W$ with " -1 " on the right-to-left diagonal corresponds to the network topology shown in Figure 2 where two node pairs are competing for the shared resources. The symbol "-1" means that these node pairs are inhibiting each other (so trying to influence competitor in the way that it would get less resources). Matrix $W$ with "1" on the right-to-left diagonal corresponds to the network topology similar to that shown in Figure 1; node pairs do not share any resources and try to activate each other to ensure optimal routing and router utilization.

In this paper two systems of differential equations will be considered. Each system consists of two differential equations. This means that the networks consist of two node pairs. First, we look at the network similar to depicted in Figure 1 with matrix $W$ consisting of " 1 "-entries on the right-to-left diagonal. Then we deal with the network similar to described in Figure 2 with matrix $W$ that contains "- 1 "-entries on the right-to-left diagonal.

\section{The system}

The dynamics of the expression level of the protein on the i-th gene, $x_{i}$, is described as

$$
\frac{d x_{i}}{d t}=f\left(\sum_{j=1}^{n} W_{i j} x_{j}-\Theta\right) v_{g}-x_{i} v_{g}+\eta
$$


The first and second terms at the right hand side represent the deterministic behavior of gene $i$, and the third term $\eta$ represents stochastic behavior. The deterministic behavior controls the $x_{i}$ due to the effects of activation and inhibition from the other genes. Those regulations of protein expression levels on gene $i$ by other genes are indicated by regulatory matrix $W_{i j}$, which takes -1 , 0 , or +1 , corresponding to activation, no regulatory interaction, and inhibition of the $i$-th gene by the $j$-th gene.

The rate of increase in the expression level is given by the sigmoidal regulation function,

$$
f(z)=\frac{1}{1+e^{-\mu z}}, \quad \text { where } \quad z=\Sigma W_{i j} x_{j}-\Theta
$$

$\Theta$ is a regulatory parameter which can be adjusted, and $\mu$ indicates the gain parameter of the sigmoidal function. The growth rate parameter $v_{g}$ represents the conditions of the metabolic reaction network [9].

\section{Two-dimensional system. First case}

We consider first the system

$$
\left\{\begin{array}{l}
\frac{d x_{1}}{d t}=\frac{1}{1+\exp \left(-\mu\left(W_{11} x_{1}+W_{12} x_{2}-\Theta\right)\right)} \cdot v_{g}-v_{g} x_{1} \\
\frac{d x_{2}}{d t}=\frac{1}{1+\exp \left(-\mu\left(W_{21} x_{1}+W_{22} x_{2}-\Theta\right)\right)} \cdot v_{g}-v_{g} x_{2}
\end{array}\right.
$$

where $W=\left(\begin{array}{ll}W_{11} & W_{12} \\ W_{21} & W_{22}\end{array}\right)=\left(\begin{array}{cc}0 & 1 \\ 1 & 0\end{array}\right)$.

Set $v_{g}=1$. The system (3.1) becomes

$$
\left\{\begin{aligned}
\frac{d x_{1}}{d t} & =\frac{1}{1+e^{-\mu\left(x_{2}-\Theta\right)}}-x_{1} \\
\frac{d x_{2}}{d t} & =\frac{1}{1+e^{-\mu\left(x_{1}-\Theta\right)}}-x_{2}
\end{aligned}\right.
$$

where $\mu$ and $\Theta$ are positive parameters.

Our goal is to study the phase portrait and the attracting sets of this system.

The standard analysis of a phase portraits includes the following stages:

- detecting critical points;

- linearization of a system around the critical points;

- making conclusions about types of the critical points;

- visualization.

In presence of parameters the analysis is more complicated. Properties of the system and the number of critical points and their location and their types can change significantly. Therefore bifurcations (essential changes in properties of the system) are to be studied also. This is our goal in the sequel. 


\subsection{Critical points}

The critical points of system (3.2) are solutions $\left(x_{1}, x_{2}\right)$ of the system

$$
\left\{\begin{array}{l}
\frac{1}{1+e^{-\mu\left(x_{2}-\Theta\right)}}-x_{1}=0, \\
\frac{1}{1+e^{-\mu\left(x_{1}-\Theta\right)}}-x_{2}=0,
\end{array}\right.
$$

or

$$
\left\{\begin{array}{l}
x_{1}=\frac{1}{1+e^{-\mu\left(x_{2}-\Theta\right)}}, \\
x_{2}=\frac{1}{1+e^{-\mu\left(x_{1}-\Theta\right)}} .
\end{array}\right.
$$

Since both right hand sides in (3.3) are positive but less than unity, all critical points locate in the square $(0,1) \times(0,1)$.

Proposition 1. All critical points of system (3.3) are of the form $(x, x)$.

Proof. Denote $\varphi(y)=\frac{1}{1+e^{-\mu(y-\Theta)}}$. The derivative $\varphi^{\prime}(y)>0$ for any positive $\mu$ and $\Theta$. It follows from (3.3) that $x_{2} \varphi\left(x_{2}\right)=x_{1} \varphi\left(x_{1}\right)$ for critical points. Since the function $y \varphi(y)$ is strictly increasing (one has that the derivative $\left.\varphi(y)+y \varphi^{\prime}(y)>0\right)$ any critical point lies on the bisectrix $x_{1}=x_{2}$.

How many critical points the system may have? In Fig. 3 the dependence of the $x$-coordinate of a critical point on the parameter $\Theta$ is shown for various values of the second parameter $\mu$. It follows that for $\mu \in(0,4)$ there is exactly one critical point; for $\mu \in(0,+\infty)$ it is possible for some range of $\Theta$ that there are exactly three critical points; the situation for $\mu=4$ is specific and will be explained in the sequel.

\subsection{Linearized system}

The linearized system around a possible critical point $\left(x_{1}, x_{2}\right)$ is

$$
\left\{\begin{aligned}
u^{\prime} & =-u+\frac{\mu e^{-\mu\left(x_{2}-\Theta\right)}}{\left[1+e^{-\mu\left(x_{2}-\Theta\right)}\right]^{2}} \cdot v \\
v^{\prime} & =\frac{\mu e^{-\mu\left(x_{1}-\Theta\right)}}{\left[1+e^{-\mu\left(x_{1}-\Theta\right)}\right]^{2}} \cdot u-v
\end{aligned}\right.
$$

It follows from (3.3) that

$$
e^{-\mu\left(x_{2}-\Theta\right)}=\frac{1}{x_{1}}-1, \quad e^{-\mu\left(x_{1}-\Theta\right)}=\frac{1}{x_{2}}-1 .
$$

Therefore, by (3.3) and Proposition 1, the coordinate $x$ of any critical point $(x, x)$ satisfies

$$
x=\frac{1}{1+e^{-\mu(x-\Theta)}} .
$$

Figure 3 shows that for some $\mu$ and $\Theta$ there are respectively one, two or three critical points. 


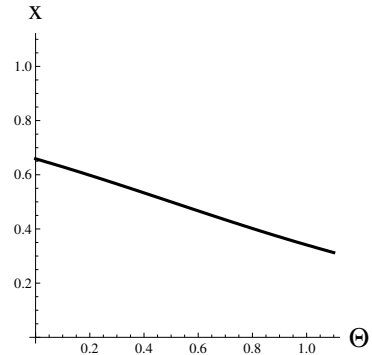

(a)

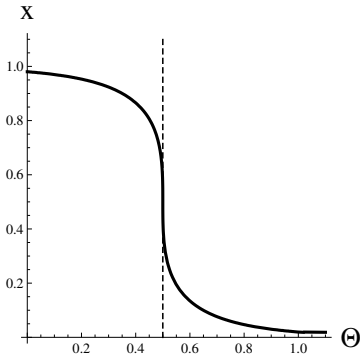

(b)

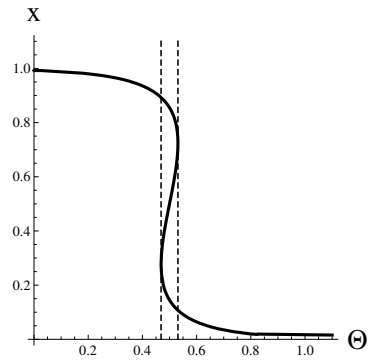

(c)

Figure 3. The dependence of $x$ of $\Theta$ for a) $\mu \in(0 ; 4)$, b) $\mu=4$ and c) $\mu \in(4 ; \infty)$, vertical dashed lines for $\Theta_{1}$ and $\Theta_{2}$.

Using (3.5), (3.6) the linearized system (3.4) can be written as

$$
\left\{\begin{array}{l}
u^{\prime}=-u+\mu x(1-x) v \\
v^{\prime}=\mu x(1-x) u-v
\end{array}\right.
$$

Let $A$ be the coefficient matrix for the system (3.7). Then

$$
\operatorname{det} A=1-\mu^{2} x^{2}(1-x)^{2}=[1-\mu x(1-x)][1+\mu x(1-x)] .
$$

Notice that $x$ depends on $\mu$ and $\Theta$. For $\mu \in(0 ; 4) \operatorname{det} A \neq 0$; for $\mu=4 \operatorname{det} A=$ 0 if $\Theta=\frac{1}{2}$ and $x=\frac{1}{2}$; for $\mu \in(4 ;+\infty) \operatorname{det} A=0$ if $\Theta=x+\frac{1}{\mu} \ln \left(\frac{1}{x}-1\right)$, where $x_{1,2}=\frac{1}{2} \pm \frac{\sqrt{\mu^{2}-4 \mu}}{2 \mu}$ (see Figure 4 ).

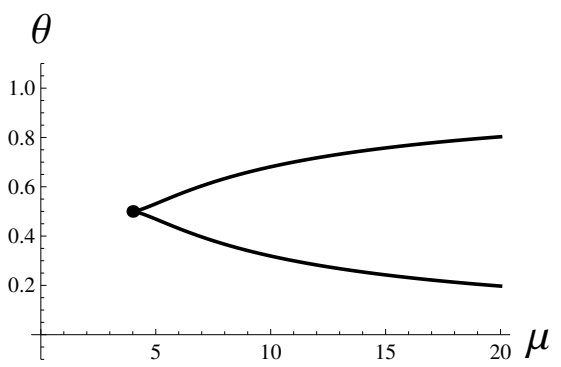

Figure 4. Two branches $\Theta=x+\frac{1}{\mu} \ln \left(\frac{1}{x}-1\right)$, where $x=\frac{1}{2}+\frac{\sqrt{\mu^{2}-4 \mu}}{2 \mu}$ (upper branch), $x=\frac{1}{2}-\frac{\sqrt{\mu^{2}-4 \mu}}{2 \mu}$ (lower branch).

The characteristic equation for (3.7) is

$$
(1+\lambda)^{2}=\mu^{2} x^{2}(1-x)^{2} .
$$

Therefore

$$
\lambda=-1 \pm \mu x(1-x) .
$$

The dependence of $\lambda$-s of $x$ and through (3.6) of $\Theta$ (for $\mu$ given) is depicted in Figure 5. 


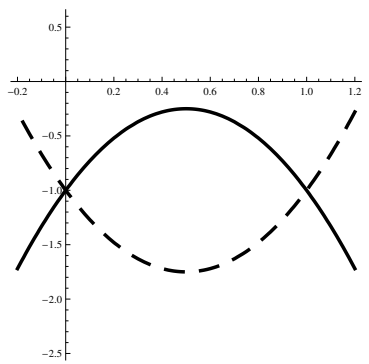

(a)

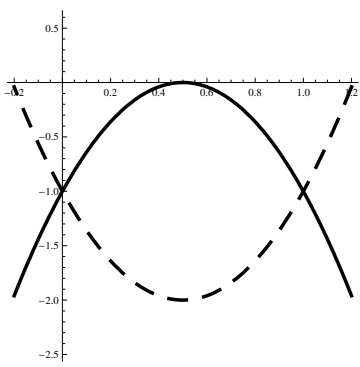

(b)

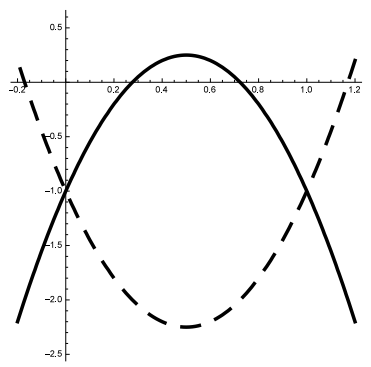

(c)

Figure 5. Roots of characteristic equation (3.8), thick line for $-1+\mu x(1-x)$, dashed line for $-1-\mu x(1-x)$, for a) $\mu \in(0 ; 4)$, b) $\mu=4$ and c) $\mu \in(4 ; \infty)$.

\section{Two-dimensional system. Second case}

Consider the system (3.1), where $W=\left(\begin{array}{ll}W_{11} & W_{12} \\ W_{21} & W_{22}\end{array}\right)=\left(\begin{array}{cc}0 & -1 \\ -1 & 0\end{array}\right)$.

The system (3.1) looks

$$
\left\{\begin{aligned}
\frac{d x_{1}}{d t} & =\frac{1}{1+e^{\mu\left(x_{2}+\Theta\right)}}-x_{1}, \\
\frac{d x_{2}}{d t} & =\frac{1}{1+e^{\mu\left(x_{1}+\Theta\right)}}-x_{2},
\end{aligned}\right.
$$

where $\mu$ and $\Theta$ are positive parameters. The critical points are solutions of the system

$$
\left\{\begin{array}{l}
x_{1}=\frac{1}{1+e^{\mu\left(x_{2}+\Theta\right)}}, \\
x_{2}=\frac{1}{1+e^{\mu\left(x_{1}+\Theta\right)}} .
\end{array}\right.
$$

It follows from (4.2) that all critical points are in the square $(0,1) \times(0,1)$. The linearized system at a critical point $\left(x_{1}, x_{2}\right)$ is

$$
\left\{\begin{array}{c}
u^{\prime}=-u-\frac{\mu e^{\mu\left(x_{2}+\Theta\right)}}{\left[1+e^{\mu\left(x_{2}+\Theta\right)}\right]^{2}} \cdot v \\
v^{\prime}=-\frac{\mu e^{\mu\left(x_{1}+\Theta\right)}}{\left[1+e^{\mu\left(x_{1}+\Theta\right)}\right]^{2}} \cdot u-v
\end{array}\right.
$$

Since

$$
e^{\mu\left(x_{2}+\Theta\right)}=1 / x_{1}-1, \quad e^{\mu\left(x_{1}+\Theta\right)}=1 / x_{2}-1
$$

the system (4.3) can be written

$$
\left\{\begin{array}{c}
u^{\prime}=-u-\mu x_{1}\left(1-x_{1}\right) v, \\
v^{\prime}=-\mu x_{2}\left(1-x_{2}\right) u-v .
\end{array}\right.
$$

Let $A$ be the coefficient matrix for the system (4.4). Then

$$
\operatorname{det} A=1-\mu^{2} x_{1} x_{2}\left(1-x_{1}\right)\left(1-x_{2}\right) .
$$


Notice that $x_{1}$ and $x_{2}$ are dependent on $\mu$ and $\Theta$.

The characteristic equation for (4.4) is

$$
(1+\lambda)^{2}=\mu^{2} x_{1} x_{2}\left(1-x_{1}\right)\left(1-x_{2}\right) .
$$

\subsection{Symmetric case}

Let us consider critical points lying on the bisectrix. All these points are of the form $(x, x)$ and $x$ can be found from (4.2)

$$
x=1 /\left(1+e^{\mu(x+\Theta)}\right) .
$$

Proposition 2. For any positive $\mu$ and $\Theta$ there is exactly one critical point of the form $(x, x)$.

Proof. The proof follows from elementary observation that in (4.6) on the left is increasing to $+\infty$ function starting at the origin and on the right there is strictly decreasing (for any positive $\mu$ and $\Theta$ ) function of $x$ which takes a positive value $\frac{1}{1+e^{\mu \Theta}}$ at $x=0$.

\subsection{Asymmetric case. Example}

In contrast to system (3.2) which may have critical points only of the form $(x, x)$ system (4.1) may have critical points also aside of the bisectrix.

Indeed, suppose that the critical point for system $(4.1)$ is of the form $(\xi, \eta)$, where $0<\xi<\eta<\frac{1}{2}$. Then, due to (4.2),

$$
\left\{\begin{array}{l}
\xi=\frac{1}{1+e^{\mu(\eta+\Theta)}}, \\
\eta=\frac{1}{1+e^{\mu(\xi+\Theta)}} .
\end{array}\right.
$$

We will find parameters $\mu$ and $\Theta$ so that for given $\xi$ and $\eta$ the system (4.7) is satisfied. It follows from (4.7) that

$$
\left\{\begin{array}{l}
e^{\mu(\eta+\Theta)}=1 / \xi-1 \\
e^{\mu(\xi+\Theta)}=1 / \eta-1
\end{array}\right.
$$

or

$$
\left\{\begin{array}{l}
\mu(\eta+\Theta)=\ln (1 / \xi-1) \\
\mu(\xi+\Theta)=\ln (1 / \eta-1)
\end{array}\right.
$$

Solving (4.8) with respect to $\mu$ and $\Theta$ one gets that

$$
\Theta=\frac{\eta \ln \left(\frac{1}{\eta}-1\right)-\xi \ln \left(\frac{1}{\xi}-1\right)}{\ln \left(\frac{1}{\xi}-1\right)-\ln \left(\frac{1}{\eta}-1\right)}, \quad \mu=\frac{1}{\eta-\xi}\left[\ln \left(\frac{1}{\xi}-1\right)-\ln \left(\frac{1}{\eta}-1\right)\right] .
$$

Since $0<\xi<\eta$ the parameter $\mu$ is positive. The parameter $\Theta$ is positive only if the inequality

$$
\eta \ln (1 / \eta-1)-\xi \ln (1 / \xi-1)>0
$$




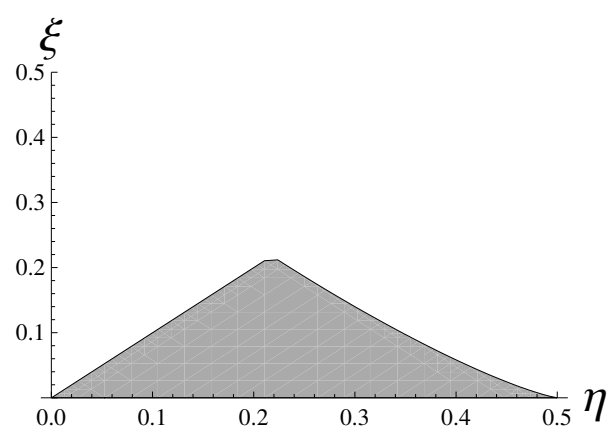

Figure 6. Region $0<\xi<\eta$, described by the inequality (4.9).

holds. For $\xi<\eta$ the region (4.9) is visualized in Figure 6.

For example, consider $\mu=20$ and $\Theta=0.07$. It follows that the system (4.7) has a solution $(0.00729,0.17569)$ (rounded for 5 digits). The symmetric point $(0.17569,0.00729)$ also solves the system. There is also a critical point $(0.06407,0.06407)$ on the bisectrix.

\section{$5 \quad$ Phase portraits for system (3.2)}

Consider the particular cases of $\mu=5$. Let us detect the signs of $\lambda=-1 \pm$ $5 x(1-x)$. The smallest $\lambda=-1-5 x(1-x)$ always is negative (dashed curve in Figure 5). The larger $\lambda=-1+5 x(1-x)$ (solid curve) is positive for $x \in\left(\frac{1}{2}-\frac{\sqrt{5}}{10}, \frac{1}{2}+\frac{\sqrt{5}}{10}\right)$. The type of critical point depends on $x$. Therefore for

$$
x \in\left(0 ; \frac{1}{2}-\frac{\sqrt{5}}{10}\right) \bigcup\left(\frac{1}{2}+\frac{\sqrt{5}}{10} ; 1\right)
$$

the critical point is a stable node (both $\lambda$ are negative and different). For

$$
x \in\left(\frac{1}{2}-\frac{\sqrt{5}}{10} ; \frac{1}{2}+\frac{\sqrt{5}}{10}\right)
$$

the critical point is the saddle point (two $\lambda$ have different signs).

There exist $\Theta_{1}=\frac{1}{2}-\frac{\sqrt{5}}{10}+\frac{1}{5} \ln \frac{3+\sqrt{5}}{2}$ and $\Theta_{2}=\frac{1}{2}+\frac{\sqrt{5}}{10}+\frac{1}{5} \ln \frac{3-\sqrt{5}}{2}$ (depicted in Figure 3) such that for $\Theta \in\left(0, \Theta_{1}\right)$ there is one critical point, for $\Theta=\Theta_{1}$ there are two critical points, for $\Theta \in\left(\Theta_{1}, \Theta_{2}\right)$ there are three critical points, for $\Theta=\Theta_{2}$ there are two critical points, and for $\Theta>\Theta_{2}$ there is one critical point.

\subsection{Particular case I}

In the first particular case the parameter $\Theta=0.3$ is from the interval $\left(0, \Theta_{1}\right)$. The system (3.2) has one critical point $(0.965332 ; 0.965332)$. According to (5.1) the type of critical point is a stable node. The respective phase portrait is depicted in Figure 7a. 


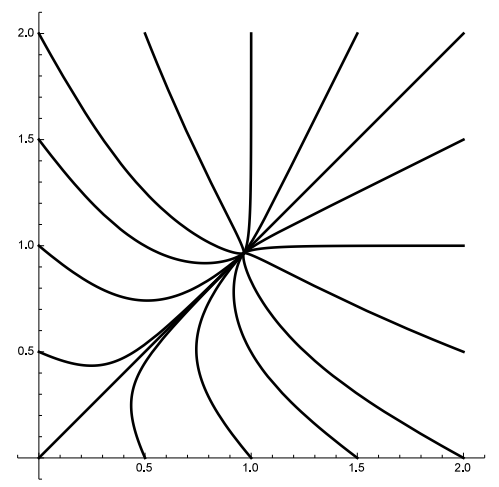

(a) $\mu=5 ; \Theta=0.3$.

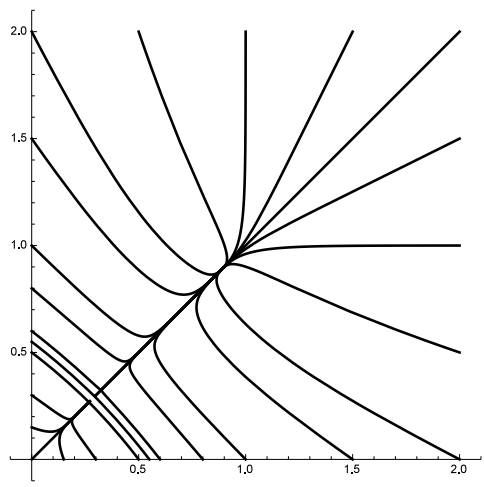

(b) $\mu=5 ; \Theta=\frac{1}{2}-\frac{\sqrt{5}}{10}+\frac{1}{5} \ln \frac{3+\sqrt{5}}{2}$.

Figure 7. The phase portraits with different parameters.

\subsection{Particular case II}

In the second particular case the parameter $\Theta=\Theta_{1}=\frac{1}{2}-\frac{\sqrt{5}}{10}+\frac{1}{5} \ln \frac{3+\sqrt{5}}{2}$. The system (3.2) has first critical point $\left(\frac{1}{2}-\frac{\sqrt{5}}{10} ; \frac{1}{2}-\frac{\sqrt{5}}{10}\right)$ of degenerate type and the second critical point $(0.89279 ; 0.89279)$ of the type node according to (5.1). The linearized system (3.7) at the first critical point becomes

$$
\left\{\begin{array}{l}
u^{\prime}=-u+v \\
v^{\prime}=u-v
\end{array}\right.
$$

and the coefficient matrix is degenerate. One has that $u^{\prime}+v^{\prime}=0$ and therefore $u+v=c$, where $c$ is an arbitrary constant. The respective phase portrait is depicted in Figure 7b.

\subsection{Particular case III}

In the third particular case the parameter $\Theta=0.5$ is from the interval $\left(\Theta_{1}, \Theta_{2}\right)$. The system (3.2) has two critical points $(0.144794 ; 0.144794)$ and $(0.855206 ; 0.855206)$ of the type node according to $(5.1)$ and the one critical point $(0.5 ; 0.5)$ of the type saddle point according to $(5.2)$. The respective phase portrait is depicted in Figure 8a.

\subsection{Particular case IV}

In the fourth particular case the parameter $\Theta=\Theta_{2}=\frac{1}{2}+\frac{\sqrt{5}}{10}+\frac{1}{5} \ln \frac{3-\sqrt{5}}{2}$. The system (3.2) has first critical point $(0.10721 ; 0.10721)$ of the type node according to $(5.1)$ and the second critical point $\left(\frac{1}{2}+\frac{\sqrt{5}}{10} ; \frac{1}{2}+\frac{\sqrt{5}}{10}\right)$ of degenerate type that can be treated exactly as the previous degenerate case. The respective phase portrait is depicted in Figure 8b. 


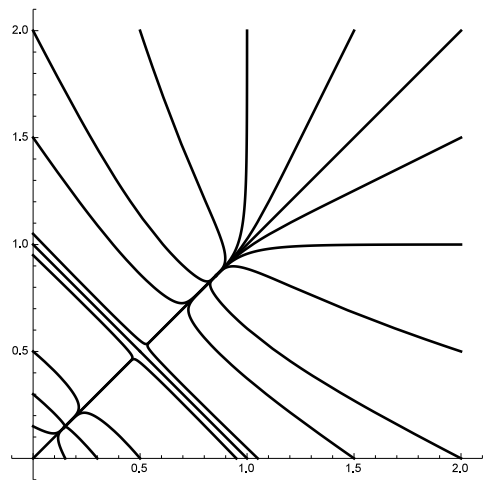

(a) $\mu=5 ; \Theta=0.5$.

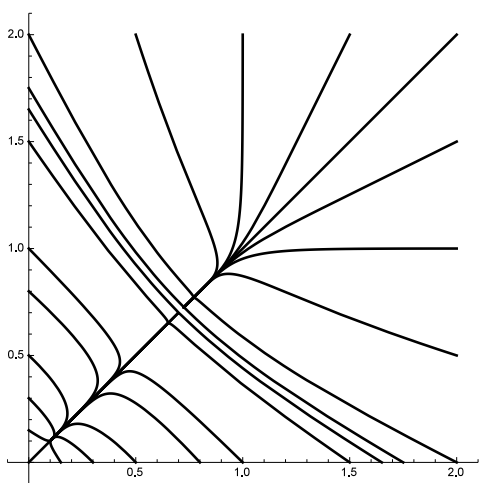

(b) $\mu=5 ; \Theta=\frac{1}{2}+\frac{\sqrt{5}}{10}+\frac{1}{5} \ln \frac{3-\sqrt{5}}{2}$.

Figure 8. The phase portraits with different parameters.

\subsection{Particular case $\mathrm{V}$}

In the fifth particular case the parameter $\Theta=1$ is from the interval $\left(\Theta_{2},+\infty\right)$. The system (3.2) has one critical point $(0.00692709 ; 0.00692709)$ of the type node according to (5.1). The respective phase portrait is depicted in Figure 9a.

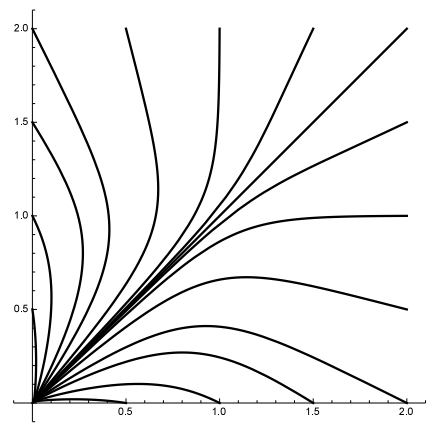

(a) $\mu=5 ; \Theta=1$.

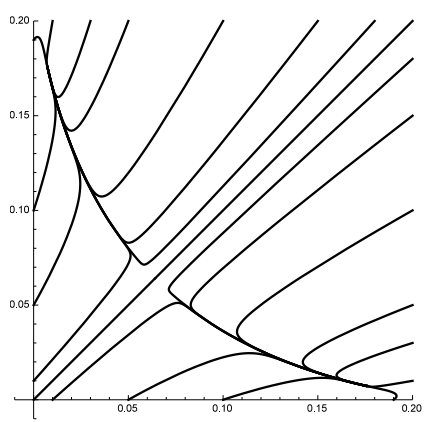

(b) The phase portrait for asymmetric case considered in subsection $4.2(\mu=20 ; \Theta=0.07)$. There are three critical points and two of them (stable nodes) are aside of the bisectrix.

Figure 9. The phase portraits with different parameters.

\section{$6 \quad$ Phase portrait for system (4.1)}

Consider the particular cases of $\mu=20, \Theta=0.07$. The type of critical point $(0.06407,0.06407)$ is saddle point (one $\lambda$ is positive and the other $\lambda$ is negative). The types of critical points $(0.00729,0.17569)$ and $(0.17569,0.00729)$ can be 
detected calculating $\lambda$ in (4.5). It follows that $\lambda \approx-1 \pm 0.647476$ and both are negative. Therefore the types of both critical points aside of bisectrix are stable nodes. The respective phase portrait is depicted in Figure $9 \mathrm{~b}$.

\section{Conclusions}

In this paper the system (3.1) is considered under the simplifying condition $v_{g}=1$. The regulatory matrices are $W_{11}=W_{22}=0, W_{12}=W_{21}=1$ and $W_{11}=W_{22}=0, W_{12}=W_{21}=-1$.

In the first case analysis shows that for $\mu \in(0,4)$ for any positive $\Theta$ there is one critical point of the type "stable node". For $\mu=4$ and for all positive $\Theta$, except the special value $\Theta=0.5$ there is exactly one critical point of the same type. For $\mu>4$ there are two values of $\Theta, \Theta_{1}<\Theta_{2}$ with the property: if $\Theta \in$ $\operatorname{ext}\left[\Theta_{1}, \Theta_{2}\right]$, then there is one critical point "stable node"; if $\Theta \in \operatorname{int}\left[\Theta_{1}, \Theta_{2}\right]$, then there are two critical points of the type "stable node" and one saddle point between them; if $\Theta=\Theta_{1}$ or $\Theta=\Theta_{2}$ then there are two critical points, one of the type "stable node" and another one attracting degenerate point (the matrix of coefficients of the linearized system is degenerate with the zero determinant). The phase portraits are visualized for various $\Theta$ provided that $\mu=5$. On these phase portraits the system has exactly one attracting stable node for $0<\Theta<\Theta_{1}=\frac{1}{2}-\frac{\sqrt{5}}{10}+\frac{1}{5} \ln \frac{3+\sqrt{5}}{2}$. The system has two stable nodes and one attracting-repelling saddle point in-between them for $\Theta \in\left(\Theta_{1}, \Theta_{2}\right)$, where $\Theta_{2}=\frac{1}{2}+\frac{\sqrt{5}}{10}+\frac{1}{5} \ln \frac{3-\sqrt{5}}{2}$. For greater $\Theta$ there is also one attracting stable node. There are two special values of $\Theta$, namely $\Theta_{1}$ and $\Theta_{2}$, for which the attractor consists of a stable node and a degenerate attracting critical point.

In the second case there is always a (unique) critical point of the form $(x, x)$. In the considered example it is a saddle point. It is possible that there exist also critical points of the form $\left(x_{1}, x_{2}\right)$ aside the bisectrix. Then the coordinates $x_{1}$ and $x_{2}$ have to satisfy the condition (4.9). In the example the type of both "out of bisectrix" critical points is a stable node. Therefore the structure of attracting sets for both cases may differ essentially.

As to the beginning of the article and telecommunications and gene networks the following should be said. It is useful to model telecommunications networks by means of the theory of gene regulatory ones.

Gene regulatory networks are characterized by high adaptivity to rapid and/or unpredictable changes. In the adaptivity process the important role is played by attractors of elements of network. For the very particular case considered the structure of attractors is simple, namely they consist of a number of critical points. The number of critical points, their location and their character may vary along with the change of parameters as was described above.

\section{References}

[1] F.M. Alakwaa. Modeling of gene regulatory networks: A literature review. J. of Computaional Systems Biology, 1:8 pages, 2014. 
[2] G. Agrawal and D. Medhi. Lightpath topology configuration for wavelengthrouted IP/MPLS networks for time-dependent traffic. Proceedings of GLOBECOM, pp. 1-5, 2006. http://dx.doi.org/10.1109/glocom.2006.35.

[3] D. Finaskins and G. Lauks. Attractor selection mechanism simulink model. Proc. 2014 Federated Conference on Computer Science and Information Systems, ACSIS, 3:71-77, 2014. http://dx.doi.org/10.15439/2014F83.

[4] N. Vijesh, S.K. Chakrabarti and J. Sreekumar. Modeling of gene regulatory networks: A review. J. Biomedical Science and Engineering, 6:223-231, 2013. http://dx.doi.org/10.4236/jbise.2013.62A027.

[5] C. Furusawa and K. Kaneko. A generic mechanism for adaptive growth rate regulation. PLoS Computational Biology, 4(1):e3, 2008. http://dx.doi.org/10.1371/journal.pcbi.0040003.

[6] F. Ricciato, S. Salsano, A. Belmonte and M. Listanti. Off-line configuration of a MPLS over WDM network under time-varying offered traffic. Proceedings of IEEE INFOCOM, 1:57-65, 2002. http://dx.doi.org/10.1109/infcom.2002.1019246.

[7] Y. Koizumi, T. Miyamura, S. Arakawa, E. Oki, K. Shiomoto and M. Murata. Robust virtual network topology control based on attractor selection. Proceedings of ONDM, pp. 123-128, 2009.

[8] Y. Koizumi, T. Miyamura, S. Arakawa, E. Oki, K. Shiomoto and M. Murata. Application of attractor selection to adaptive virtual network topology control. 3d International ICST Conference on Bio-Inspired Models of Network, Information, and Computing Systems, (5), 2010. http://dx.doi.org/10.4108/ICST.BIONETICS2008.4714.

[9] Y. Koizumi, T. Miyamura, S.I. Arakawa, E. Oki, K. Shiomoto and M. Murata. Adaptive virtual network topology control based on attractor selection. Journal of Lightwave Technology, 28(11):1720-1731, 2010. http://dx.doi.org/10.1109/JLT.2010.2048412.

[10] A. Lakhina, K. Papagiannaki, M. Crovella, C. Diot, E.D. Kolaczyk and N. Taft. Structural analysis of network traffic flows. Proceedings of ACM Sigmetrics, 28:61-72, 2004. http://dx.doi.org/10.1145/1005686.1005697.

[11] B. Chen, G.N. Rouskas and R. Dutta. On hierarchical traffic grooming in WDM networks. IEEE/ACM Transactions on Networking, 16(5):1226-1238, 2008. http://dx.doi.org/10.1109/TNET.2007.906655.

[12] H. de Jong. Modeling and simulation of genetic regulatory systems: A literature review. J. of Computaional Biology, 9(1):67-103, 2002. http://dx.doi.org/10.1089/10665270252833208. 